\title{
EXCELÊNCIA NO GERENCIAMENTO DE CONSTRUÇÃO DE HIDRELÉT'RICA DE GRANDE PORTE
}

\section{CONSTRUCTION MANAGEMENT EXCELENCE OF MAJOR HYDROELECTRIC POWER PLANTS}

\section{ANTONIO OKABAYASHI}

Mestre em Administração pelo Centro Universitário Nove de Julho (Uninove).

Professor da Universidade São Judas Tadeu. Rua Vuturuna, 169, Saúde - São Paulo - SP - CEP 04147-080

E-mail: aokabayashi@gmail.com

\section{MARCOS ALBERTO CASTELHANO BRUNO}

Doutor em Administração pela Faculdade de Economia, Administração e Contabilidade da Universidade de São Paulo (FEA/USP). Professor visitante na Università Luigi Bocconi (Itália). Pesquisador associado da Faculdade de Economia, Administração e Contabilidade da Universidade de São Paulo (FEA/USP). Avenida Prof. Luciano Gualberto, 908, sala E-1 11, Cidade Universitária São Paulo - SP - CEP 05508-900

E-mail:marcos.acbruno@gmail.com

ROBERTO SBRAGIA

Doutor em Administração pela Faculdade de Economia, Administração e Contabilidade da Universidade de São Paulo (FEA/USP).

Professor da Faculdade de Economia, Administração e Contabilidade da Universidade de São Paulo (FEA/USP). Avenida Prof. Luciano Gualberto, 908, sala E-117, Cidade Universitária São Paulo - SP - CEP 05508-900

E-mail: rsbragia@usp.br 


\section{RESUMO}

Este trabalho verifica a aplicação das práticas gerenciais descritas no PMBOK, na gestão da construção de duas hidrelétricas de grande porte no Brasil. Utilizando o método do estudo de caso, esta pesquisa permitiu a obtenção dos dados práticos referentes a 23 proposições selecionadas do PMBOK. O levantamento de campo, realizado em 2005 , ocorreu por observação direta, coleta de documentos, entrevistas estruturadas e abertas. Visando à obtenção de dados sob perspectivas diferentes, foram selecionados para entrevista não só os gestores atuantes nos canteiros de obras dos dois projetos, mas também os executivos da matriz e um consultor. Os resultados atestam que as práticas de gerenciamento adotadas pelos gestores dos dois projetos, caso objeto deste estudo, são aderentes às proposições do PMBOK. Obteve-se também a descrição das práticas de gerenciamento de tempo, custo, comunicação e outras práticas relevantes não contempladas pelo modelo PMBOK. O trabalho permite recomendar que os referenciais relativos a caminho crítico, orçamento e flexibilização das proposições do PMBOK estão entre os aspectos prioritários a serem investigados em futuras pesquisas sobre gerenciamento de projetos.

\section{PALA VRAS-CHAVE}

Gerenciamento de projetos; Excelência em projeto; Construção de hidrelétrica; PMBOK; Projetos complexos.

\section{ABSTRACT}

This study verifies the application of the management practices proposed in the PMBOK to the construction management of two major hydroelectric power plants in Brazil. Using the case study method, this research has allowed to assess management pratices concerning twenty-three PMBOK's selected propositions. The direct observations, the documents surveys and the interviews - using the 
multiple-choice and the constructed-response questions - were the methods used to collect the required data and evidences. The job site managers, the executives of the head office and the consultant were interviewed so as to get different views of the information gathered. The research results attest that the management practices applied by managers in both studied projects are adherents to the PMBOK propositions. Moreover, it was possible to describe management practices related to time, cost, communication and to others relevant practices that were taken for granted by $\mathrm{PMBOK}$. This study indicates that the references related to the project critical path, the project budget and the flexibility of the propositions of the PMBOK are among of prior aspects to be investigate in the future researches about project management.

\section{KEYWORDS}

Project management; Project excellence; Hydroelectric power plant construction; PMBOK; Complex projects.

\section{INTRODUÇÃO}

As grandes realizações históricas como a construção das muralhas chinesas, das pirâmides egípcias e dos teatros gregos e a viagem de Cristóvão Colombo guardam similaridade com as realizações modernas, como a viagem tripulada à Lua com retorno seguro, a de Almir Klink ao Pólo Sul, a construção da Hidrelétrica de Itaipu e a do Eurotúnel. Essa similaridade se estende também às realizações internas das empresas modernas, como desenvolvimento e implantação de novos sistemas de informação, desenvolvimento de novas estruturas organizacionais e redesenho de processos produtivos para adequação às novas tecnologias e atendimento aos objetivos estratégicos. A similaridade entre essas realizações, tão diferentes entre si, que ocorrem em locais e tempos diversos, é o caráter único e singular. O sucesso dessas realizações singulares exige a aplicação de disciplinas, ferramentas, técnicas, sistemas e principalmente a atuação de pessoas dotadas de habilidades para combinação de conhecimentos, administração de conflitos, solução de problemas e outros desafios peculiares de cada realização. Na teoria da administração, a disciplina pertinente ao estudo dessas realizações singulares é o gerenciamento de projetos.

Trata-se de um tema de interesse das comunidades internacionais de profissionais. O principal representante destas é o Project Management Institu- 
te (PMI), responsável pela emissão do compêndio Project management body of knowledge (PMBOK), que hoje atinge cerca de um milhão de exemplares nas versões oficiais, em sete línguas. No Brasil, há capítulos do PMI em São Paulo, em Belo Horizonte, em Curitiba, em Manaus e no Rio de Janeiro (CLELAND; IRELAND, 2002; PROJECT MANAGEMENT INSTITUTE, 2004).

A importância do gerenciamento da construção de hidrelétricas no Brasil pode ser avaliada pela relevância da energia elétrica de origem hidráulica. Noventa por cento da energia elétrica consumida no Brasil é de origem hidráulica, e o potencial hidráulico a explorar do País é de cerca de $75 \%$ dos 270 mil MW (AGÊNCIA NACIONAL DE ENERGIA ELÉTRICA, 2005).

A atenção continuada dos especialistas e o impacto no bem-estar de quase totalidade do povo brasileiro perfazem as duas características que conferem importância ao tema (CASTRO, I978).

Assim, a verificação da aderência das práticas gerenciais adotadas na construção de usinas hidrelétricas de grande porte à metodologia de gerenciamento de projeto preconizada pelo PMI no PMBOK (PROJECT MANAGEMENT INSTITUTE, 2002), a descrição das práticas gerenciais relevantes das áreas de conhecimento, tempo, custo e comunicação, e a descrição das práticas relevantes não contempladas no PMBOK são os três objetivos desta pesquisa.

A estrutura de desenvolvimento deste artigo é composta por cinco seções, que seguem a formatação tradicional de artigos acadêmicos, a saber: introdução, revisão bibliográfica, metodologia, discussão de resultados e conclusão. A principal característica deste trabalho é a indexação dos dados e resultados segundo o sumário e a página do PMBOK, aproximando a prática da teoria.

\section{CONCEITOS E DEFINIÇÕES DO GERENCIAMENTO DE PROJETOS E O MODELO PMBOK}

Segundo a literatura afim, a conceituação de projeto engloba elementos básicos, tais como: o caráter único ou singular qualificando os produtos, os serviços, os processos ou as seqüências de atividades; o caráter temporário em que o prazo está associado a datas de início e de término; o caráter não repetitivo; a limitação do consumo de recursos, da necessidade de satisfação dos requisitos dos produtos ou serviços; o caráter exclusivo da atividade de uma empresa; o esforço de coordenação; o alcance dos objetivos; e as mudanças benéficas (CAUPIN et al., I999; KEELLING, 2002; KERZNER, 2002; MAXIMIANO, 2002; PRADO, 2003; PROJECT MANAGEMENT INSTITUTE, 2002; VARGAS, 2003). 
Outro conjunto de autores conceitua gerenciamento de projeto como o processo que envolve os seguintes aspectos, entre os mais relevantes: aplicação de conhecimentos, habilidade e ferramentas; atendimento a requisitos; produção de resultados; uso de recursos da empresa; tomada de decisões; e manutenção da motivação das pessoas nos processos de planejamento, execução e controle (ATKINSON, I999; CAUPIN et al., I999; KERZNER, 2002; KUPRENAS et al., 2000; MAXIMIANO, 2002; PROJECT MANAGEMENT INSTITUTE, 2002; VARGAS, 2003).

Vargas (2003), Cleland e Ireland (2002), Maximiano (2002) e Project Management Institute (2002) descrevem a equipe de projeto inserida em uma organização segundo uma graduação diferente de autonomia. Wilemon (I998) destaca as estruturas inovadoras das organizações do projeto, rompendo os limites de uma organização. Kloppenborg e Opfer (2002) enfatizam a padronização dos processos, o uso da internet e o gerenciamento das comunicações e de riscos como as tendências na área de conhecimento de gerenciamento de projetos.

A despeito de grande quantidade de estudos envolvendo pesquisadores de reconhecida autoridade no assunto, os projetos continuam falhando. Na sua análise, Keeling (2002) observa que apenas 29\% dos projetos concluídos satisfazem, em sua plenitude, os componentes de sucesso: conceito, objetivos, qualidade do resultado, custo e tempo. Dinsmore (I992) propôs diminuir as falhas estabelecendo mandamentos destinados a gerentes de projetos e Prado (200I) na linha semelhante desenvolveu uma lista de verificação para identificação de fatores críticos de sucesso de projetos com base em depoimentos dos profissionais de gerenciamento de projeto. Kerzner (2002) estendeu o conceito de sucesso de um projeto, incluindo, entre outros fatores, a aceitação do cliente e o alinhamento estratégico a custo, prazo e qualidade.

Outros estudiosos examinaram o sucesso dos projetos relacionando-o à capacidade de lidar com a complexidade dos projetos. Nesse sentido, Willians (I999) destaca, entre outros aspectos, a transferência de risco de tempo para o contratado e a exigência de redução de prazo de entrega do projeto como fatores que determinam a complexidade do projeto. $\mathrm{O}$ autor defende a necessidade de aprimoramento de habilidade da equipe de projeto no uso combinado de ferramentas como engenharia simultânea com as práticas tradicionais. Taxén e Lilliesköld (2008), acreditando que as ferramentas tradicionais como PERT/ CPM, EAT e Gráfico de Gant têm amplitude limitada, privilegiando o controle, propõem lidar com a crescente complexidade dos projetos usando ferramentas alternativas para gerenciamento de projeto, com visão mais ampla, porém sem perder o foco para ação. 


\section{FigURA I}

\section{GRUPO DE PROCESSOS DE GERENCIAMENTO DE PROJETOS DO PMBOK}

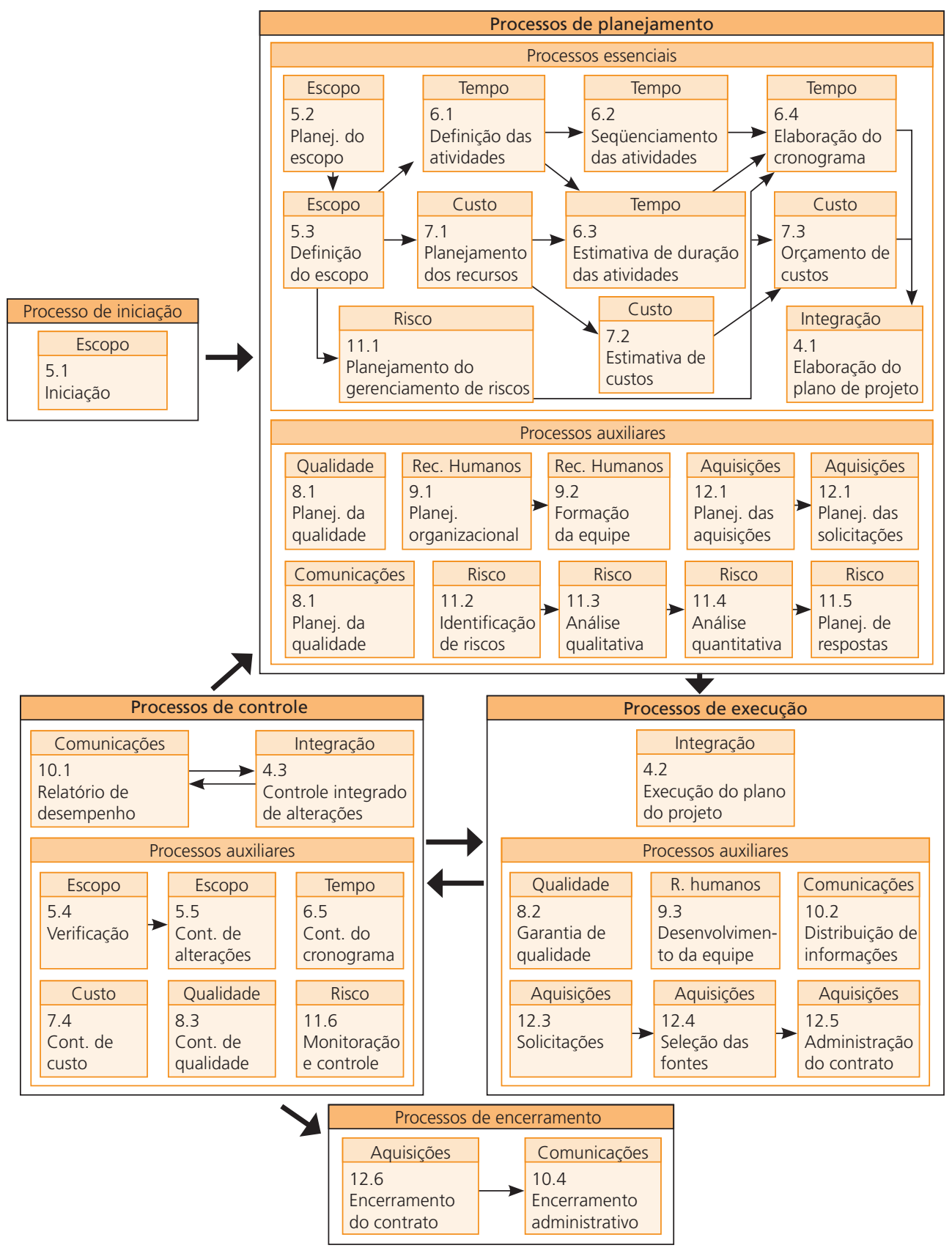

Fonte: Okabayashi (2005). 
As melhores práticas das diversas linhas de estudo, com objetivos de alcance do sucesso dos projetos, estão hoje consolidadas na forma de manuais ou compêndios, entre os quais o PMBOK do PMI e o Projects in controlled environments (PRINCE2) do Office of Government Commerce (OGC) do Reino Unido.

A Figura I é uma representação das inter-relações dos 39 processos do PMBOK.

Os quadros menores, contidos nos cinco quadros de títulos sombreados, representam os processos das nove áreas de conhecimento: integração, escopo, tempo, custo, qualidade, recursos humanos, comunicação, risco e aquisição. Os agrupamentos dos processos iniciação, planejamento, execução, controle e execução são representados nos quadros de títulos sombreados.

A OGC exige a aplicação do PRINCE2 a todos os seus prestadores de serviço (SIEGELAUB, 2008).

Na comparação com o PMBOK, Siegelaub (2008) entende que o PRINCE2 é prescritivo e privilegia o formalismo e controle. Kerzner (2002) acredita que o gerenciamento informal pode levar à conclusão com sucesso de um projeto, desde que os elementos básicos, como confiança, comunicação, cooperação e trabalho em equipe, façam parte da cultura da organização.

\section{APRESENTAÇÃO do CASO E CONTEÚdo DA PESQUISÁ}

A empresa onde o estudo foi realizado é paulista, faturou no ano de $2004 \mathrm{um}$ total de R\$ I, 6 bilhão e empregou I2.600 pessoas. Construiu, até 2004, dezoito hidrelétricas, responsáveis pela geração de $39.900 \mathrm{MW}$.

Os dois projetos que compõem o objeto de estudo desta pesquisa são a construção de duas hidrelétricas brasileiras. São similares e devem gerar juntas I. $570 \mathrm{MW}$, ambas com prazo contratual de 54 meses, dotadas de alojamentos para atender 3.000 pessoas e a volumes médios de serviços de enrocamento, concretagem e montagem, respectivamente, de $12.000 .000 \mathrm{~m}^{3}, 300.000 \mathrm{~m}^{3} \mathrm{e}$ I2.000 t. A empresa e os consórcios representantes dos clientes mostravam-se satisfeitos com os resultados.

As fases características das etapas de construção de hidrelétrica são: mobilização e instalação de canteiro de obras; escavação e terraplenagem; construção de estrutura de concreto; montagem eletromecânica; comissionamento; desmontagem de instalações e desmobilização.

Durante a realização do estudo, as equipes de comissionamento estavam em franca mobilização em ambas as hidrelétricas, embora as duas estivessem na fase final de construção e com o pequeno contingente remanescente em redução. 
As atividades de levantamento de dados basearam-se em: entrevistas abertas, entrevistas estruturadas construídas em escala de Likert, com gradação para cinco níveis de percepção, observações diretas e evidências documentais. Essas quatro fontes de evidências para este estudo de caso único conferem a qualidade adequada à pesquisa. Optou-se por estudo de caso único, por tratar-se de uma rara oportunidade de observação da aplicação do modelo PMBOK em construção simultânea de hidrelétricas de grande porte, por uma mesma empresa e na mesma região do Brasil. Essa opção está em conformidade com um dos fundamentos conceituais do método do estudo de caso proposto por Yin (200I), que reconhece a validade da adoção do caso único para fenômenos com características muito específicas.

A observação direta foi realizada no canteiro de obra dos dois projetos e registraram-se observações das instalações dos dois canteiros e de três reuniões. Foram entrevistados onze profissionais, sendo seis gestores atuantes nos dois projetos, um superintendente, dois diretores e o presidente na matriz, além de um consultor externo. Obteve-se, assim, visão sob diversas perspectivas. Aplicou-se para os onze profissionais uma entrevista aberta, e para os seis gestores dos projetos, uma entrevista adicional com questões estruturadas. As entrevistas foram gravadas com anuência dos entrevistados.

A entrevista estruturada foi concebida para abranger os trinta e nove processos das nove áreas de conhecimento distribuídas nos cinco agrupamentos dos processos do modelo do PMBOK. Optou-se pela formulação de vinte e três questões distribuídas nos agrupamentos de processos: planejamento, execução e controle. A quantidade de questões foi limitada pela duração máxima das entrevistas, estipulada em noventa minutos, e na definição da abrangência nos agrupamentos de processos prevaleceu a consideração da fase de construção das hidrelétricas em que se praticava pouca ou nenhuma atividade de iniciação e encerramento.

As questões e as evidências documentais associadas receberam a mesma numeração do sumário do PMBOK e da página em que é desenvolvido o assunto neste compêndio. Todos os entrevistados receberam previamente um exemplar do PMBOK para superar as dificuldades de nomenclatura. A obtenção dos resultados das entrevistas abertas baseou-se em matriz de respondente e assunto, obtida por meio da leitura das transcrições aprovadas das entrevistas gravadas (BAUER; GASKELL, 2002). Os resultados das observações diretas ficaram registrados na própria ferramenta de observação.

O critério de qualidade para avaliar o grau de confiabilidade dos dados foi: a prática deve ser sustentada por pelo menos três fontes de evidências e a resposta ao questionário estruturado deve ser enquadrada no grau maior da escala de Likert por cinco dos seis respondentes. 


\section{RESULTADOS E DISCUSSÃO}

\subsection{SÍNTESE DAS EVIDÊNCIAS E CRITÉRIO DA QUALIDADE DOS DADOS}

Nesta seção, analisam-se a qualidade dos dados, quanto ao grau de atendimento ao critério estabelecido para este estudo, e a aderência de cada uma das vinte e três práticas gerenciais que foram pesquisadas às proposições do $\mathrm{PMBOK}$. O Quadro I contém as evidências encontradas.

\section{QUADRO I}

SÍNTESE DAS EVIDÊNCIAS ENCONTRADAS

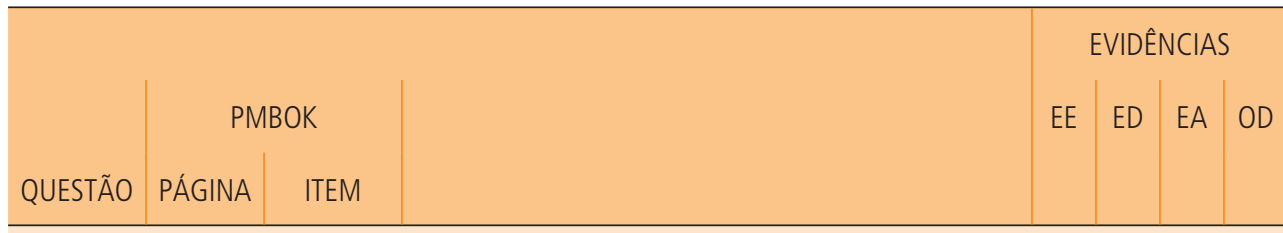

PLANEJAMENTO

\begin{tabular}{|c|c|c|l|c|c|c|c|}
\hline A & 56 & 05.2 .3 .1 & Objetivos do projeto & 6 & Ok & Ok & Ok \\
\hline B & 57 & 05.3 .2 .1 & EAT Estrutura analítica do trabalho & 6 & Ok & Ok & Ok \\
\hline C & 67 & 06.1 .2 .2 & Lista de atividades & 5 & Ok & Ok \\
\hline D & 69 & 06.2 .2 .1 & Diagrama de precedência & 4 & Ok & \\
\hline E & 73 & 06.3 .2 .3 & Duração com base quantitativa & 5 & Ok & & Ok \\
\hline F & 76 & 06.4 .2 .5 & Software: gerenciamento de projeto - tempo & 6 & Ok & Ok & Ok \\
\hline G & 86 & 07.1 .2 .3 & Software: gerenciamento de projeto - custo & 5 & Ok & Ok & \\
\hline H & 88 & 07.2 .2 .3 & Estimativa de baixo para cima dos custos & 5 & Ok & Ok & \\
\hline I & 90 & 07.3 .3 .1 & Base de referência dos custos & 5 & Ok & Ok & \\
\hline J & 101 & 08.1 .3 .3 & Listas de verificação & 6 & Ok & Ok & Ok \\
\hline K & 111 & 09.1 .3 .1 & Matriz de designação de responsabilidades & 5 & Ok & Ok & \\
\hline L & 120 & 10.1 .2 .1 & Análise dos interessados & 5 & Ok & Ok & Ok \\
\hline M & 135 & 11.3 .2 .2 & Matriz de probabilidade e impacto & 6 & Ok & \\
\hline
\end{tabular}




\section{QUADRO I (CONTINUAÇÃO)}

\section{SÍNTESE DAS EVIDÊNCIAS ENCONTRADAS}

\begin{tabular}{|c|c|c|c|c|c|c|c|}
\hline \multirow[b]{3}{*}{ QUESTÃO } & \multirow{2}{*}{\multicolumn{2}{|c|}{ PMBOK }} & & \multicolumn{4}{|c|}{ EVIDÊNCIAS } \\
\hline & & & & \multirow[t]{2}{*}{$\mathrm{EE}$} & \multirow[t]{2}{*}{ ED } & \multirow[t]{2}{*}{ EA } & \multirow[t]{2}{*}{ OD } \\
\hline & PÁGINA & ITEM & & & & & \\
\hline \multicolumn{8}{|c|}{ EXECUÇÃO } \\
\hline $\mathrm{N}$ & 47 & 04.2 .2 .3 & Sistema de autorização de trabalho & 5 & Ok & Ok & Ok \\
\hline $\mathrm{O}$ & 101 & 08.2 .2 .2 & Auditorias de qualidade & 6 & Ok & Ok & Ok \\
\hline$P$ & 115 & 09.3 .2 .3 & Sistemas de reconhecimento e premiação & 6 & Ok & Ok & Ok \\
\hline Q & 121 & 10.2.2.2 & Sistemas de recuperação de informação & 6 & Ok & Ok & Ok \\
\hline $\mathrm{R}$ & 159 & 12.5.2.2 & Relatório de desempenho do fornecedor & 6 & Ok & Ok & Ok \\
\hline \multicolumn{8}{|c|}{ CONTROLE } \\
\hline$S$ & 80 & 06.5 .2 .5 & Análise dos desvios & 6 & Ok & Ok & Ok \\
\hline $\mathrm{T}$ & 92 & 07.4 .3 .4 & Estimativa na conclusão & 6 & Ok & Ok & \\
\hline$U$ & 103 & 08.3 .2 .3 & Diagrama de Pareto & 0 & & & \\
\hline V & 123 & 10.3.2.4 & Análise do trabalho realizado & 5 & Ok & Ok & \\
\hline$X$ & 145 & 11.6.2.2 & Revisões periódicas dos riscos do projeto & 4 & Ok & Ok & \\
\hline
\end{tabular}

Legenda:

EE - Entrevista estruturada: o número da coluna corresponde à quantidade de gestores que responderam que praticam e usam sistematicamente a proposição.

ED - Evidência documental: Ok significa que o entrevistado apresentou documentos que confirmam a prática.

EA - Entrevista aberta: Ok significa que as respostas das questões abertas confirmam a prática.

OD - Observação direta: Ok significa que as observações diretas confirmam a prática.

As colunas "Página" e "Item" foram incluídas para facilitar a recorrência ao PMBOK.

Fonte: Okabayashi (2005).

Os dados contidos nas quatro linhas sombreadas não atendem ao critério da qualidade. Dezenove proposições do PMBOK têm uso sistemático confirmado, seus dados atendem ao critério da qualidade especificado para este trabalho, e suas práticas, quando confrontadas com a descrição respectiva da proposição do PMBOK (indicadas nas colunas "Página" e "Item" do PMBOK no Quadro I), apresentam-se aderentes. Apenas o diagrama de Pareto, comprovadamente, não é praticado por nenhum entrevistado. 


\subsection{DESCRIÇÃO DAS PRÁTICAS RELEVANTES DO GERENCIAMENTO DO TEMPO, DOS CUSTOS E DAS COMUNICAÇÕES DOS PROJETOS}

Os dados obtidos e a verificação da consistência permitem descrever as práticas relevantes do gerenciamento do tempo, dos custos e das comunicações adotadas nos dois projetos. A prática das principais ferramentas contidas nas proposições do PMBOK para essas três áreas de conhecimento foi evidenciada e analisada com auxílio da Figura I.

No gerenciamento do tempo, a reunião semanal de prazo, que é a prática da análise dos desvios preconizada pelo $\mathrm{PMBOK}$, apoiada pelos processos de elaboração, de revisão, de emissão de diversas versões e de controle de cronograma, é o ponto alto do gerenciamento de tempo praticado nos dois projetos.

No gerenciamento dos custos, as reuniões de cobrança do indicador comparativo de custo real com o orçamento, nos diversos níveis hierárquicos - incluindo os colaboradores braçais - mostram-se com freqüência adequada para cada nível, sendo apoiadas nos processos de elaboração, de revisão, de emissão nas suas diversas versões de orçamento e custo, e constituem as práticas culturais marcantes identificadas no gerenciamento dos custos nos dois projetos.

No gerenciamento das comunicações do projeto, a disciplina na realização das diversas reuniões previstas no plano de comunicação (entendendo por disciplina nas reuniões a pontualidade, o ritual, a freqüência e o comportamento) e a disponibilidade das informações na alimentação dessas reuniões (incluindo reuniões com os colaboradores braçais e os painéis de comunicação visual) são os pontos altos do gerenciamento de comunicações praticados nos dois projetos. Os processos corporativos de captação, tratamento e acumulação de informação apóiam essas atividades.

\subsection{PRÁTICAS, PERCEPÇÕES E RELATOS RELEVANTES NÃO CONTEMPLADOS NA VERSÃO DO PMBOK ADOTADA COMO REFERÊNCIA}

Desdobramento da estratégia e célula de trabalho são duas práticas que permitem o alinhamento dos objetivos e das metas do projeto com o planejamento estratégico. O primeiro destina-se a atender aos níveis gerenciais e a segunda inclui o grande contingente de trabalhadores braçais de permanência curta e programada. A cerimônia de reconhecimento que homenageia os integrantes das células que atingem os resultados é praticada com visível entusiasmo, gerando um ambiente contagiante, propício para a formação do espírito de equipe. 
O gerenciamento de segurança e o da preservação do meio ambiente são componentes fortes da cultura da empresa. Mostram-se especialmente críticos no gerenciamento da construção de hidrelétricas por causa da particularidade da atividade que expõe o trabalhador braçal e o meio ambiente a riscos potencialmente elevados. Os seus indicadores são obrigatórios no elenco de indicadores das células e das reuniões mensais de análise de desempenho. A prática do gerenciamento de segurança é visível em todos os níveis hierárquicos dos dois projetos, principalmente pela avaliação diária do nível de segurança do projeto, medido por um indicador resultante de um jogo entre os trabalhadores braçais e os gerentes. Os primeiros denunciam situações de risco a que estão submetidos por falta de planejamento, e os demais apontam atos inseguros praticados pelos trabalhadores braçais. A prática do gerenciamento da preservação do meio ambiente é visível no planejamento da instalação de instrumentos de coleta e encaminhamento de resíduos e de efluentes gerados no processo produtivo para tratamentos recomendados.

Nas observações diretas, registraram-se várias ações resultantes do gerenciamento da comunidade constituída pelos funcionários dos projetos, quais sejam: refeitório, alojamento, lavanderia, serviço de correio, área de lazer, templo ecumênico, sala de cinema, serviço de transporte e academia de ginástica. Todas essas ações estão, em maior ou menor intensidade, voltadas à manutenção da ordem e disciplina entre os quase dois mil operários em atividade, alojados em cada um dos projetos. O piso cerâmico branco nos refeitórios, por exemplo, pelo fato de, por si só, trazer sensação de limpeza aos seus freqüentadores, neles cultiva e inspira conduta de higiene e respeito ao local. Uma questão digna de nota é o templo ecumênico com cultos programados para diversas religiões.

Projetos com a magnitude de usinas hidrelétricas causam impactos de igual grandeza não só no meio ambiente, mas também na vida social local. Assim, há preocupação quanto a possíveis intervenções de grupos contrários à construção de barragens e, ainda, na divulgação transparente das atividades da construção. A inclusão da comunidade, principalmente das crianças, dá-se pelas visitas abertas em fins de semana ou nos dias especiais. Nessas oportunidades, realizam-se atos educativos, como plantio de árvores e passeios nas áreas degradadas em recuperação e nas usinas de triagem de lixo e de compostagem. 


\section{CONCLUSÕES, LIMITAÇÕES E RECOMENDAÇÕES}

\subsection{CONCLUSÕES}

As conclusões deste trabalho estão voltadas para a aplicabilidade do padrão de excelência em gestão contido na metodologia PMBOK, dentro do propósito de contribuir para o incremento dos conhecimentos afins, especialmente nas ligações entre a teoria e prática no gerenciamento de projeto.

Os resultados deste estudo puderam atestar que dezenove das vinte e três proposições descritas no PMBOK, objetos de análise deste trabalho, são comprovadamente aplicadas no gerenciamento dos dois projetos. Ficou evidenciado também que as práticas são aderentes às respectivas proposições daquele compêndio.

No que diz respeito à descrição das três práticas relevantes do gerenciamento de tempo, custo e comunicação, contempladas pelo modelo PMBOK, observouse que as práticas de gerenciamento de tempo e custo estão disseminadas em todos os níveis hierárquicos da empresa. Constituem fatores decisivos para essa disseminação os instrumentos disponibilizados no gerenciamento das comunicações, baseados em sistemas computadorizados, reuniões sistemáticas e equipes treinadas e disciplinadas. Acrescente-se a aplicação da estrutura analítica do trabalho comum aos processos que permite estabelecer as bases para a consistência entre o cronograma e o orçamento.

O desdobramento da estratégia e a célula de trabalho se mostram adequados para o gerenciamento de projetos complexos de grande porte, especialmente no relacionamento com grande contingente de mão-de-obra, com pouca especialização. O gerenciamento da segurança, da preservação do meio ambiente, dos interessados da comunidade e do apoio comunitário são áreas de interesse particular da construção civil de grande porte. Essas duas conclusões constituem os destaques das observações obtidas por meio da descrição das práticas relevantes não contempladas no PMBOK.

São ainda pertinentes as considerações que se seguem, a título de conclusões gerais.

Os preceitos do PMBOK combinados com a aprendizagem da empresa formam um conjunto de boas práticas para o gerenciamento de projetos da complexidade e magnitude da construção de uma hidrelétrica.

O êxito dos projetos e a constatação de aplicação das ferramentas relevantes permitem inferir que as práticas gerenciais dos dois projetos são aderentes ou estão em nível de desenvolvimento muito próximo de aderência aos preceitos propostos no PMBOK. 


\subsection{LIMITAÇÕES}

Como típico de um estudo de caso, o presente estudo apresenta algumas limitações. As principais são: (a) estudo de um único caso, que limita a possibilidade de inferência dos resultados para além do caso estudado; (b) quantidade limitada de visitas aos projetos, reduzindo as oportunidades de observação de processos produtivos e reuniões de avaliação de custos e de avaliação de desempenho; (c) possíveis vieses nas respostas dos entrevistados, decorrentes de situações de pressão, indisponibilidade de informação precisa e outros fatores associados. Essas limitações são reconhecidas e justificáveis neste trabalho e devem ser superadas em estudos posteriores.

\subsection{RECOMENDAÇÕES}

No tocante às proposições do modelo do $\mathrm{PMBOK}$, dois aspectos podem ser objeto de recomendação para desenvolvimento de investigação futura: a possibilidade de flexibilização do formalismo, considerando a proposição do Kerzner (2002), e novas posturas comportamentais dos gestores do projeto quanto aos referenciais relativos a caminho crítico e orçamento. Esta segunda recomendação decorre da percepção havida durante os trabalhos desta pesquisa de que esses dois referenciais podem tornar-se limitações indesejáveis para os gestores, que podem ser levados a cumprir as referências, temporal e financeira, sem refletir sobre eventuais alternativas aos caminhos indicados, deixando de empregar e desenvolver criatividade. Nessa linha de investigação, as contribuições de Taxén e Lilliesköld (2008) podem ser frutíferas no que diz respeito ao uso de ferramentas alternativas que privilegiam as ações.

Do ponto de vista prático, podem-se recomendar estudos que se voltem para os seguintes aspetos: alinhamento dos objetivos do projeto aos objetivos corporativos, na medida em que esta pesquisa corroborou essa necessidade já detectada por Stewart (200I), alinhamento das ações do grande contingente de trabalhador braçal de curta permanência programada, atendimento das necessidades comunitárias e inserção do projeto no ambiente de comunidade existente.

\section{REFERÊENCIAS}

AGÊNCIA NACIONAL DE ENERGIA ELÉTRICA. Atlas de energia elétrica do Brasil. 2. ed. Brasília: Aneel, 2005.

ATKINSON, R. Project management: cost, time and quality, two best guesses and a phenomenon, its time to accept other success criteria. International Journal of Project Management, Guildford, v. I7, n. 6, p. 337-342, Dec. I999. 
BAUER, M. W.; GASKELL, G. Pesquisa qualitativa com texto, imagem e som: um manual prático. Tradução Pedrinho A. Guareschi. Petrópolis: Vozes, 2002.

CASTRO, C. M. A prática da pesquisa. São Paulo: McGraw-Hill, I978.

CAUPIN, G. et al. ICB international project management association: competence baseline. Bremen: Eigenverlag, I999.

CLELAND, D. I.; IRELAND, L. R. Gerência de projetos. Rio de Janeiro: Reichmann \& Affonso, 2002. DINSMORE, P. C. Gerência de programas e projetos. São Paulo: Pini, I992.

KEELLING, R. Gestão de projetos: uma abordagem global. São Paulo: Saraiva, 2002.

KERZNER, H. Gestão de projetos: as melhores práticas. Porto Alegre: Bookman, 2002.

KLOPPENBORG, T. J.; OPFER, W. A. Forty years of project management research: trends, interpretation, and predictions. In: PINTO, J. K.; CLELAND, D. I.; SLEVIN, D. P. The frontier of project management research. Newtown Square: PMI, 2002. p. 3-30.

KUPRENAS, J. A. et al. Project manager workload-assessment of values and influences. Project Management Journal, Drexel Hill, v. 3I, n. 4, p. 44-5I, Dec. 2000.

MAXIMIANO, A. C. A. Administração de projeto: como transformar idéias em resultados. 2. ed. São Paulo: Atlas, 2002.

OKABAYASHI, A. Gestão da construção de hidrelétrica de grande porte: aderência de práticas gerenciais ao modelo PMBOK - ênfase em tempo, custo e comunicação. 2005. 225 f. Dissertação (Mestrado)-Centro Universitário Nove de Julho, São Paulo, 2005.

PRADO, D. Planejamento e controle de projetos. 4. ed. Belo Horizonte: Desenvolvimento Gerencial, 200I (Série Gerência de Projetos, v. 2).

Gerenciamento de projetos nas organizações. 2. ed. Belo Horizonte: Desenvolvimento Gerencial, 2003 (Série Gerência de Projetos, v. I).

PROJECT MANAGEMENT INSTITUTE. PMBOK Guide: um guia do conjunto de conhecimentos do gerenciamento de projetos, edição 2000. Newtown Square: PMI, 2002.

PROJECT MANAGEMENT INSTITUTE. PMI 2004 annual report. Newtown Square: PMI, 2004. SIEGELAUB, J. M. How PRINCE2 can complement PMBOK and your PMP. Disponível em: <http:// www.prince2.com/prince2-downloads.asp>. Acesso em: 3I jul. 2008.

STEWART, W. Balanced scorecard for projects. Project Management Journal, Drexel Hill, v. 32, n. I, p. 38- 53, Mar. 200I.

TAXÉN, L.; LILLIESKÖLD, J. Images as action instruments in complex projects. International Journal of Project Management, Guildford, v. 26, n. 5, p. 527-536, Jul. 2008.

VARGAS, R. Gerenciamento de projetos: estabelecendo diferenciais competitivos. 5. ed. Rio de Janeiro: Brasport, 2003 .

WILEMON, D. Cross-functional cooperation. In: PINTO, J. K. (Ed.). The Project Management Institute: project management handbook. San Francisco: Jossey-Bass, I998. p. 279-299.

WILLIANS, M. The need for new paradigms for complex projects. International Journal of Project Management, Guildford, v. I7, n. 5, p. 269-273, Oct. I999.

YIN, R. K. Estudo de caso: planejamento e método. 2. ed. Porto Alegre: Bookman, 200I. 